\title{
Effectiveness of Dry Needling versus Placebo on Gait Performance, Spasticity, Electromyographic Activity, Pain, Range-of-Movement and Quality of Life in Patients with Multiple Sclerosis: A Randomized Controlled Trial Protocol
}

\author{
Carlos Luque-Moreno ${ }^{1,2}$ (), Anabel Granja-Domínguez ${ }^{2}$, Jose A. Moral-Munoz ${ }^{1,3, * \mathbb{C}}$, \\ Guillermo Izquierdo-Ayuso ${ }^{2}$, David Lucena-Anton ${ }^{1}[$ and Alberto Marcos Heredia-Rizo 4 (i) \\ 1 Department of Nursing and Physiotherapy, University of Cadiz, 11009 Cadiz, Spain; \\ carlos.luque@uca.es (C.L.-M.); david.lucena@uca.es (D.L.-A.) \\ 2 DINAC Foundation, Vithas Nisa Hospital, 41950 Seville, Spain; \\ anabel.granja@fundaciondinac.com (A.G.-D.); guillermo.izquierdo@fundaciondinac.com (G.I.-A.) \\ 3 Institute of Research and Innovation in Biomedical Sciences of the Province of Cadiz (INiBICA), \\ University of Cadiz, 11009 Cadiz, Spain \\ 4 Departamento de Fisioterapia, Facultad de Enfermería, Fisioterapia y Podología, Universidad de Sevilla, \\ 41009 Sevilla, Spain; amheredia@us.es \\ * Correspondence: joseantonio.moral@uca.es
}

Received: 18 November 2020; Accepted: 16 December 2020; Published: 17 December 2020

\begin{abstract}
Dry needling (DN) is an emerging technique commonly used in neurological and musculoskeletal pain conditions, but there have been no previous studies in patients with multiple sclerosis (pwMS). This trial aims to assess the efficacy of deep DN, compared with sham placebo DN, on gait performance, spasticity level, pain, electromyographic activity, range-of-movement (ROM) and quality of life in pwMS. Forty adults with MS were randomly assigned to one study group. The DN group will undergo 2 sessions (once per week) using DN over the rectus femoris (RF) and gastrocnemius medialis (GM) muscles at the lower extremity with higher spasticity. The placebo group will receive the same protocol using a sham placebo needle (Dong Bang needle). Outcome measures will include gait performance, using the GaitRite ${ }^{\circledR}$ system, spasticity level with the Modified Ashworth Scale, superficial electromyographic activity of RF and GM, pain (pressure algometer), ROM (goniometer), and quality of life (Musiqol). This study is the first investigating the short-term effect of DN, compared with placebo, in pwMS, and taking into account the possible changes in the electromyographic activity of the lower limb. Therefore, the results may help to understand the suitability of using this technique in the clinical setting for this population. Trial registration: ACTRN12619000880145.
\end{abstract}

Keywords: multiple sclerosis; gait disorders; neurologic; pain management; muscle spasticity; physical therapy modalities; dry needling; clinical trial protocol

\section{Introduction}

Multiple sclerosis (MS) is one of the most common and disabling neurologic conditions among early adults, affecting approximately 2.3 million people worldwide [1], and with a higher prevalence in women (3:1 ratio) [2]. The specific cause of the disease is still uncertain, and thus the individual course and prognosis are difficult to predict [3]. Persons with MS (pwMS) suffer from varying cognitive and sensory-motor impairments that affect their functional capacity, including impaired gait performance [4]. This is reported as a major complaint in up to $85 \%$ of pwMS [5], and seems to be 
closely related to increased fall risk [6]. Chronic pain, especially at the lower extremities (LE), is also a very disabling symptom within this population, with an overall prevalence of $50 \%$ [7]. Spasticity and muscle weakness are present from the initial stages of the disease and have been associated with abnormal gait patterns [8], which may worsen other symptoms and impact on the quality of life (QOL). For example, the presence of spasticity contributes to modifying the speed, cadence, stride length, and swing phase during gait, and to a lower hip and knee range-of-movement (ROM) [9]. Additionally, walking-related motor fatigue in pwMS is attributed to the higher proportion of force that LE muscles, especially the soleus and gastrocnemius muscles, have to generate during prolonged walking [10].

The clinical management of MS-related disorders includes pharmacological and non-pharmacological interventions. In severely disabled patients, rehabilitation aims to rebalance the altered metabolic and muscle parameters [11], and the main goal is to improve functional independence and QOL [12]. Overall, physiotherapy treatments can be categorized into four domains: physical training (fitness, endurance, or resistance training), neuroproprioceptive facilitation or inhibition, motor skill acquisition, also called guided individualized therapy, and technology-based interventions [13]. However, the evidence for most approaches is still limited for the management of aspects such as walking capability [12], spasticity level [14], and self-reported pain intensity [15,16]. In addition, the recommendations about the necessity of improving the access to treatment for pwMS, to adapt the interventions to the individual disability level, and to use up-to-date evidence-based approaches do not seem to be properly implemented in the clinical setting $[17,18]$.

Dry needling (DN) is a novel but commonly used technique for patients with musculoskeletal pain, although there is conflicting evidence about its effectiveness [19,20]. In people with central nervous system disorders, the efficacy of DN has been scarcely investigated in stroke survivors, with purported positive effects on spasticity, pain, and ROM [21-23]. Even though DN is mostly a safe technique, minor adverse events, e.g., bruising, bleeding, and pain, are common [24], and need to be considered.

The specific effect of DN, when compared with sham placebo DN, has been barely assessed in people with neurological conditions [25]. Patients' expectations regarding the treatment may influence the results and evoke changes associated with the placebo effect. In fact, it has been suggested that the patient-practitioner relationship may be enough to improve the QOL and the results after intervention in some patients [26], although there is certain discrepancy about this topic [27]. In chronic musculoskeletal disorders, there are no definite conclusions on the superiority of using real deep DN over placebo DN [28]. Therefore, more studies that compare these two approaches are needed to better understand this issue.

To our knowledge, no previous randomized controlled trials have investigated the effects of using deep DN in pwMS. Recently, our research group reported promising findings in a clinical case study following the same protocol [29]. DN aims to induce a localized stretch of the soft tissue and may help to modulate the motoneuron activity and decrease the excitability of spinal reflexes, even though the exact physiological mechanisms that support this technique remain unknown [30]. The use of DN also seems to optimize the results of rehabilitation protocols. For example, in people with fibromyalgia, DN has been proposed as a suitable complementary approach to improve pain and QOL [31,32]. However, it is still necessary to continue evaluating the potential clinical effects of DN in many other conditions [30]. We hypothesize that using deep DN over the rectus femoris (RF) and Gastrocnemius Medialis (GM) muscles, which are directly related to the functionality of the LE, may help to evoke motor control changes that improve gait performance in pwMS. Therefore, our aim is to analyze the short-term effects of deep DN, compared to sham placebo DN, on gait parameters, spasticity level, electromyographic activity, pain intensity, ROM, and QOL in adults with MS. 


\section{Materials and Methods}

\subsection{Study Design}

This is a protocol for a placebo-controlled, double-blind, superiority, randomized clinical trial. The study protocols follows the guidelines of the SPIRIT Statement [33]. This study protocol was approved by the Andalusian Biomedical Research Ethics Committee 04/2019 (CEI Hospitales Universitarios Virgen Macarena-Virgen del Rocío, Seville, Spain) and was registered on the Australian New Zealand Clinical Trials Registry (ACTRN12619000880145).

\subsection{Setting and Eligibility Criteria}

The data will be collected in the Multiple Sclerosis Research and Treatment Unit, Dinac Foundation, Vithas Nisa Hospital, Castilleja de la Cuesta, Seville, Spain. Eligibility criteria are as follows: patients with a diagnosis of MS according to the McDonald 2010 criteria [34]; males and females older than 18 years; ability to walk for at least $2 \mathrm{~min}$ (with or without support), which represents an Expanded Disability Status Scale (EDSS) [35] score between 0-6.5; absence of MS outbreaks in the previous month; having signed the informed consent form; and being able to comply with the study protocol. Exclusion criteria are: pregnancy; patients with comorbidity signs in the last month; having received corticosteroid treatment within the previous month; inability to walk due to physical impairments; and having received botulinum toxin injections at the LE in the last 3 months.

\subsection{Procedure}

The flowchart of the study process is shown in Figures 1 and 2. At a first appointment, a neurologist will carry out a complete neurological evaluation of each patient to assess for eligibility. Data collection will be carried out at baseline (first visit) and between 6 and 8 days after the intervention protocol by a researcher (evaluator) who will be blinded to participants' allocation group. The intervention in both groups will be performed by another senior physiotherapist, who is experienced in the use of DN.

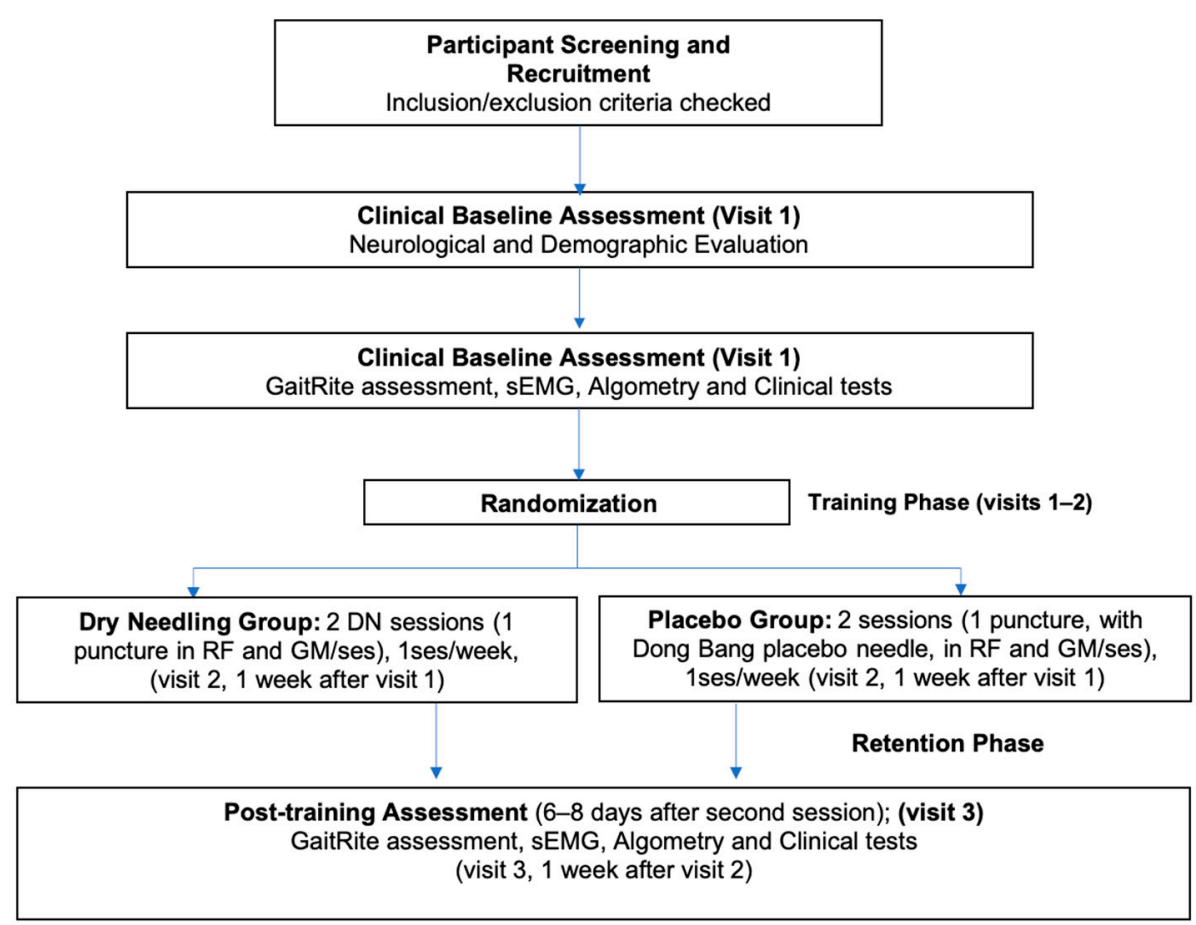

Figure 1. Study design flowchart. GM: Gastrocnemius medialis; RF: Rectus femoris; sEMG: surface Electromyography. 


\begin{tabular}{|c|c|c|c|c|c|}
\hline \multirow[b]{3}{*}{ TIMEPOINT } & \multicolumn{5}{|c|}{ STUDY PERIOD } \\
\hline & \multirow{2}{*}{$\begin{array}{c}\text { Enrollment } \\
\text { Visit } 1\end{array}$} & \multirow{2}{*}{$\begin{array}{c}\text { Allocation } \\
\text { Visit } 1\end{array}$} & \multicolumn{2}{|c|}{ Intervention } & \multirow{2}{*}{$\begin{array}{c}\begin{array}{c}\text { Post- } \\
\text { intervention }\end{array} \\
\text { Visit } 3\end{array}$} \\
\hline & & & Visit 1 & Visit 2 & \\
\hline \multicolumn{6}{|l|}{ ENROLLMENT: } \\
\hline \multirow[b]{3}{*}{ Neurological evaluation } & $\mathrm{X}$ & & & & \\
\hline & $\mathrm{X}$ & & & & \\
\hline & $\mathrm{x}$ & & & & \\
\hline Randomized Allocation & & $\mathrm{X}$ & & & \\
\hline \multicolumn{6}{|l|}{ INTERVENTIONS: } \\
\hline \multirow[b]{2}{*}{ Sham Dry Needling } & & & $\mathrm{X}$ & $\mathrm{X}$ & \\
\hline & & & $\mathrm{X}$ & $\mathrm{X}$ & \\
\hline \multicolumn{6}{|l|}{ ASSESSMENTS: } \\
\hline \multirow{2}{*}{$\begin{array}{c}\text { Social demographics } \\
\text { Primary outcomes }\end{array}$} & $\mathrm{X}$ & & & & \\
\hline & $\mathrm{X}$ & & & & $\mathrm{X}$ \\
\hline Secondary outcomes & $\mathrm{X}$ & & & & $\mathrm{X}$ \\
\hline
\end{tabular}

Figure 2. SPIRIT recommended content for the schedule of enrolment, interventions, and assessments.

The lead researcher (physical therapist) will get the signed informed consent forms, where the study details will be clearly explained, and the patients will be informed that his/her participation is voluntary so they can withdraw at any moment with no need to provide any specific reason.

\subsection{Interventions}

All patients will continue receiving their usual care during the study protocol. In addition, the DN interventions in both groups will be carried out in the Research and Treatment Unit in Multiple Sclerosis, Dinac Foundation, at Vithas Nisa Hospital, Seville, Spain.

For those in the DN group, the intervention will consist of using deep DN with disposable stainless-steel needles $(0.3 \mathrm{~mm} \times 50 \mathrm{~mm}$ or $0.3 \mathrm{~mm} \times 40 \mathrm{~mm}$, Agupunt, Barcelona, Spain), depending on the depth of the muscle to be treated. The fast in-fast out Hong technique will be used over the active or latent myofascial trigger points of the RF and GM muscles, following the diagnostic criteria and intervention guidelines previously established [36]. A minimum of 3 to 4 local twitch responses will have to be elicited. The participants' level of tolerance will be considered, and participants will be able to request to stop the treatment at any moment if unpleasant or very painful sensations appear. The intervention will be performed in the LE with higher spasticity, as expressed by the Modified Ashworth Scale score in the targeted muscles. A single puncture per muscle will be made each session. DN will be performed during 45-60 s for muscle until the local twitch responses disappeared or until the patient can no longer bear the pain. The intervention will last for 2 weeks, 1 session per week.

For the placebo group, a simulation needle procedure using a Dong Bang placebo needle, similar to the Streitberger needle, will be used. These needles appear to be effective placebo techniques in studies using acupuncture [37]. The physical therapist will follow the same treatment protocol than in the DN group. The placebo needles evoke mechanical stimulation without piercing the skin; hence, patients experience a pressure sensation similar to that of a "real" needle. 


\subsection{Outcomes}

Clinical assessment of gait performance, spasticity level, electromyographic activity, pain, ROM, and QOL will be reported in the first and third visit (Figure 2). Several outcome measures will be collected to get a better understanding of the treatment effect and because of their extensive use in research on these topics. This will allow us to compare our results with those reported in previous studies. Following the guidelines of the International Classification of Functioning, Disability and Health [38], some of the outcomes will be devoted to investigate functional parameters, such as gait parameters and QOL. The outcome measures and the tools to assess them are detailed below:

\subsubsection{Gait Performance}

The GaitRite ${ }^{\circledR}$ system will be used to measure several gait parameters (average gait speed, walking cadence, step length, stride length, step width, and percentage of support-oscillation) [39,40]. During the overground walking assessment, participants will walk at preferred speed along an $8.62 \mathrm{~m}$ walkway (the length of the tapestry). Data about gait parameters will be collected with motion sensors placed directly in the tapestry.

The two-minutes walking test (2MWT) [41] will be used to assess functional capacity. Given the difficulty that many patients with MS have to walk for $6 \mathrm{~min}$, we propose the 2MWT in the present protocol. The 2MWT is valid in pwMS and provide an efficient and alternative to the 6MWT.

\subsubsection{Spasticity}

The Modified Ashworth scale (MAS) [42] will be used to assess the spasticity level over the RF and GM muscles. The reliability of the MAS has been demonstrated in LE muscles, e.g., knee extensors and plantar flexors. Thus, this scale is a valid tool for the present protocol. Even though the MAS only measures the passive component of spasticity, the electromyographic evaluation detailed below would provide some information on the spastic co-contraction phenomena during the execution of analytical movements.

\subsubsection{Electromyographic Activity}

A superficial Electromyography (sEMG) will measure the neuromuscular activity over the RF and GM muscles and their activity during gait. This outcome will be collected with a sEMG device called mDurance $[43,44]$. The sEMG sensors will be placed in the motor point of the RF and GM muscles according to the recommendations of the SENIAM protocol [45]. To obtain the Maximal Voluntary Contraction [46] for the RF muscle, patients will lie on their back with the leg off the table (hip in extension, and knee in $90^{\circ}$ flexion), in the stretch position, and the patient will be asked for a maximum muscle contraction and to keep it for $6 \mathrm{~s}$. For the GM muscle, the patient will be placed lying on his back. With the ankle in dorsal flexion, the patient will be asked to move the ankle to plantar flexion, and to keep the muscle contraction for $6 \mathrm{~s}$. Additionally, the activity of the RF and GM muscles will be monitored during gait, both during the first minute of the 2 MWT and during the GaitRite ${ }^{\circledR}$ examination. In both situations, the Root Mean Square [10] for those muscles will be measured to indirectly quantify muscle fatigue.

\subsubsection{Pain}

The pressure pain threshold, as the minimal amount of pressure where the sensation of pressure changes to pain, will be collected with a pressure algometer (Psymtec Digital Algometer M3) [47]. The measurement will be performed bilaterally at several spots: (a) over the motor point of the RF muscle and GM muscles; (b) at the head of the splint bone, considered as a control point close to the intervention sites; and (c) distally, over the upper trapezius, as a remote control location. 


\subsubsection{Range of Movement}

The passive hip, knee, and ankle ROM will be assessed using a manual standard goniometer. $\mathrm{ROM}$ is a common outcome measure in other studies about the efficacy of DN in different conditions.

\subsubsection{Quality of Life}

The Multiple Sclerosis International QoL (Musiqol) [48] questionnaire will measure changes in the QOL. This is a multi-dimensional and self-administered questionnaire, and is available in 14 languages, including Spanish. This tool is a disease-specific QOL scale that includes items related to activities of daily living and psychological aspects, among others.

\subsection{Recruitment, Allocation, and Blinding}

The patients will be recruited as they attend their usual consultations with the neurologist, where they will be proposed to take part on the study based on the fulfillment of the established eligibility criteria. Information about the project will be posted on the institutional website where the study will be carried out.

Concerning the allocation concealment procedures, an external researcher will conduct central randomization using computer-generated random numbers (www.randomizer.org), performing a simple randomization. The two lists, DN and placebo groups, comprising a total of 40 patients, will be sent by email to the physical therapist who will perform the intervention, with the randomized numbers assigned to each group. Those numbers will correspond to the recruitment order of each patient, and they will be assigned by the physical therapist in charge of the evaluation, who will remain unaware of the allocation sequence (blinded evaluation).

To ensure blinding, participants will be fitted with a mask so they will not be able to see the type of needle used. Additionally, care providers will not be allowed to access to the room where the intervention will take place, and data analysts will be blinded to participants' allocation group. All patients will be informed of their group allocation after analyzing the study findings. If real DN shows to be superior to placebo DN, participants in the placebo group will be allowed to receive the real $\mathrm{DN}$ protocol.

\subsection{Data Collection and Management}

The data will be entered directly into the computer where the patient's medical record is stored, which is placed in the facilities where the evaluation will be carried out. This process will guarantee the security of the data. For the exchange of data between researchers for further analysis, an Excel page will be sent, including only the identification number of each patient.

\subsection{Statistical Methods}

\subsubsection{Sample Size}

The G*Power version 3.1 statistical software (Heinrich-Heine-Universität Düsseldorf) was used to estimate the sample size and statistical power. According to Salom-Moreno et al. [21] the between groups difference in pressure pain sensitivity, assessed with a pressure algometer, was $39.7 \pm 79.92 \mathrm{kPa}$ (effect size $d=0.49$ ) in the anterior tibialis muscle of the affected side. Furthermore, since this previous study did not use a placebo group as a comparison group, the study of Ghannadi et al. [25] was considered to calculate the sample size according to the MAS, that showed a difference between groups of $0.75 \pm 0.82$ points (effect size $d=0.93$ ). This would require a sample size of at least 38 participants. An alpha level of 0.05 was assumed to perform the analysis. In both cases, the calculated sample size had an $85 \%$ power to detect statistically significant and clinically meaningful changes in pressure pain sensitivity and spasticity. Therefore, we considered 40 patients to be an adequate sample size for the proposed study. 


\subsubsection{Data Analysis}

Statistical Analysis will be performed by PASW STATISTIC 18 for Windows. Descriptive statistics will be calculated to show a summarized overview of the data, including frequency counts for categorical variables and central tendency and dispersion measurements for continuous variables. Means, standard deviations (SD) and 95\% confidence intervals (CI) will be calculated for continuous variables. The Kolmogorov-Smirnov test will be used to check the normality of the data. Baseline characteristics will be compared between groups to assess the adequacy of the randomization process and analyze the homogeneity of the sample. Independent $t$-test (normal distribution) or Mann-Whitney U test (non-normal distribution) for continuous data or chi-square tests of independence for categorical data will be used for this purpose. Furthermore, within groups (paired) and between groups (unpaired) pre-post intervention differences will be analyzed using the abovementioned statistical tests, considering the nature of each variable. For all the analyses, an a priori alpha level of 0.05 will be used.

\subsubsection{Oversight and Monitoring}

The research members (CLM, AGD, JAMM, and AMHR) who conceived the study design and got funding will be part of the trial steering committee. The key role of the chief investigator (CLM) is to coordinate the ongoing project. The trial steering committee will review the progress of the trial and set up the changes and adjustments needed. In case of any serious adverse events during the trial, the lead investigator (CLM) will report them to the Institutional Research Ethics Committee (Comite de Ética de la Investigación Biomédica de Andalucía, CEI Hospitales Universitarios Virgen MacarenaVirgen del Rocío, Seville, Spain) and changes, if needed, will be discussed, and addressed.

During data collection protocol, participants will be asked to report any adverse effects. Upon completion of the study, telephone follow-up will be conducted to check for any possible adverse effects after the last treatment session.

\section{Discussion}

This innovative study will evaluate the impact of DN on gait performance, spasticity level, electromyographic activity, pain, ROM, and QoL in pwMS. Including a sham placebo DN group will allow us to discuss which effects may be related to patient's expectations and the possible motor behavior adaptations due to this effect. We selected the RF and GM as the targeted muscles for their role in the functionality of the LE in this population. These two muscles are largely responsible for the so-called stiff knee pattern $[49,50]$ caused by an excessive activation of knee extensors during the pre-swing phase [50], along with weakness of the gastrocnemius muscles [49]. This pattern is especially prevalent in pwMS. As regards the treatment protocol, there is some controversy about the number of sessions required to evoke changes after DN interventions, which range from 1 to 3 sessions in most of previous research in this field [23]. In our protocol, we will assess the changes after 2 treatment sessions.

Gait performance and the electromyographic activity of RF and GM muscles have been included as outcomes to detect the electrophysiological changes that may occur at the muscular level, although the specific mechanisms of action of the DN intervention remains controversial [30]. This will allow us to investigate the motor pattern changes. This appears to be an important outcome after using an invasive technique that acts directly over the muscle tissue which could evoke changes in the gait pattern. Thus, the interpretation of the electromyographic parameters by means of maximum voluntary contraction and root mean square may inform us about changes related to muscle force and fatigue. Additionally, functional electromyographic measurements during gait will help us to understand how these changes can influence gait patterns.

One of the strengths of this protocol is the inclusion of the placebo group. The clinical results when using invasive approaches can be influenced by patients' expectations, as an adaptation associated with the placebo effect. It has been also speculated that some patients could also benefit for these 
treatments when no real invasive approaches are used. Nevertheless, there is controversy about this issue [27], especially in patients with sensory motor deficits. Recently, the effect of DN was compared with sham DN in a single study in stroke patients [25] that found a decrease in muscle spasticity and improvements in function and gait speed after three sessions. Since stroke is a neurological disorder with different clinical features than MS, a clinical trial comparing real deep DN with sham placebo DN in pwMS is of high interest. The findings of this study may determine if one of the two approaches is superior to the other, which is relevant from a clinical point of view.

$\mathrm{DN}$ is a cheap and easy to carry out technique and serious adverse events are rare. Positive findings are expected after this study. If this is the case, DN could be considered as a feasible and useful complementary intervention, within a multimodal approach, to enhance the functional recovery of pwMS during the different clinical stages of the disease.

Author Contributions: Conception of the study: C.L.-M., A.M.H.-R., A.G.-D. and J.A.M.-M. Drafting the manuscript: C.L.-M., A.M.H.-R., A.G.-D., J.A.M.-M. and D.L.-A. J.A.M.-M. will analyze and interpret (with C.L.-M.) the patient data regarding the sEMG signal. Critical review of the manuscript: C.L.-M., A.M.H.-R., A.G.-D., J.A.M.-M., D.L.-A. and G.I.-A. All authors have read and agreed to the published version of the manuscript.

Funding: This research was supported by the Ilustre Colegio Profesional de Fisioterapeutas de Andalucía (grant $n^{\circ}$ Al-04/2019), the Department of Nursing and Physiotherapy (University of Cadiz), and the DINAC Foundation (Multiple Sclerosis Research Unit, Vithas Nisa Hospital, Seville). The funders had no role in the study design, collection, analysis, and interpretation of data or manuscript writing.

Acknowledgments: The authors thank Ana Venegas-Venegas, Cristina Páramo-Camino, Aurora Alemán-Rodríguez, and Rocío Pascual-Amodeo.

Conflicts of Interest: The authors declare no conflict of interest.

\section{References}

1. Browne, P.; Chandraratna, D.; Angood, C.; Tremlett, H.; Baker, C.; Taylor, B.V.; Thompson, A.J. Atlas of Multiple Sclerosis 2013: A growing global problem with widespread inequity. Neurology 2014, 83, 1022-1024. [CrossRef]

2. Dunn, S.E.; Steinman, L. The gender gap in multiple sclerosis: Intersection of science and society. JAMA Neurol. 2013, 70, 634-635. [CrossRef]

3. Degenhardt, A.; Ramagopalan, S.V.; Scalfari, A.; Ebers, G.C. Clinical prognostic factors in multiple sclerosis: A natural history review. Nat. Rev. Neurol. 2009, 5, 672-682. [CrossRef]

4. Comber, L.; Galvin, R.; Coote, S. Gait deficits in people with multiple sclerosis: A systematic review and meta-analysis. Gait Posture 2017, 51, 25-35. [CrossRef] [PubMed]

5. Tajali, S.; Shaterzadeh-Yazdi, M.J.; Negahban, H.; van Dieën, J.H.; Mehravar, M.; Majdinasab, N.; Saki-Malehi, A.; Mofateh, R. Predicting falls among patients with multiple sclerosis: Comparison of patientreported outcomes and performance-based measures of lower extremity functions. Mult. Scler. Relat. Disord. 2017, 17, 69-74. [CrossRef]

6. Brandstadter, R.; Ayeni, O.; Krieger, S.C.; Harel, N.Y.; Escalon, M.X.; Katz Sand, I.; Leavitt, V.M.; Fabian, M.T.; Buyukturkoglu, K.; Klineova, S.; et al. Detection of subtle gait disturbance and future fall risk in early multiple sclerosis. Neurology 2020, 94, e1395-e1406. [CrossRef] [PubMed]

7. Ferraro, D.; Plantone, D.; Morselli, F.; Dallari, G.; Simone, A.M.; Vitetta, F.; Sola, P.; Primiano, G.; Nociti, V.; Pardini, M.; et al. Systematic assessment and characterization of chronic pain in multiple sclerosis patients. Neurol. Sci. 2018, 39, 445-453. [CrossRef]

8. Givon, U.; Zeilig, G.; Achiron, A. Gait analysis in multiple sclerosis: Characterization of temporalspatial parameters using GAITRite functional ambulation system. Gait Posture 2009, 29, 138-142. [CrossRef] [PubMed]

9. Pau, M.; Coghe, G.; Corona, F.; Marrosu, M.G.; Cocco, E. Effect of spasticity on kinematics of gait and muscular activation in people with Multiple Sclerosis. J. Neurol. Sci. 2015, 358, 339-344. [CrossRef] [PubMed]

10. Eken, M.M.; Richards, R.; Beckerman, H.; van der Krogt, M.; Gerrits, K.; Rietberg, M.; de Groot, V.; Heine, M. Quantifying muscle fatigue during walking in people with multiple sclerosis. Clin. Biomech. 2020, 72, 94-101. [CrossRef] 
11. Manfredini, F.; Straudi, S.; Lamberti, N.; Patergnani, S.; Tisato, V.; Secchiero, P.; Bernardi, F.; Ziliotto, N.; Marchetti, G.; Basaglia, N.; et al. Rehabilitation Improves Mitochondrial Energetics in Progressive Multiple Sclerosis: The Significant Role of Robot-Assisted Gait Training and of the Personalized Intensity. Diagnostics 2020, 10, 834. [CrossRef] [PubMed]

12. Vanbellingen, T.; Kamm, C.P. Neurorehabilitation Topics in Patients with Multiple Sclerosis: From Outcome Measurements to Rehabilitation Interventions. Semin. Neurol. 2016, 36, 196-202. [CrossRef] [PubMed]

13. Martinková, P.; Freeman, J.; Drabinová, A.; Erosheva, E.; Cattaneo, D.; Jonsdottir, J.; Baert, I.; Smedal, T.; Romberg, A.; Feys, P.; et al. Physiotherapeutic interventions in multiple sclerosis across Europe: Regions and other factors that matter. Mult. Scler. Relat. Disord. 2018. [CrossRef] [PubMed]

14. Khan, F.; Amatya, B.; Bensmail, D.; Yelnik, A. Non-pharmacological interventions for spasticity in adults: An overview of systematic reviews. Ann. Phys. Rehabil. Med. 2019, 62, 265-273. [CrossRef]

15. Amatya, B.; Young, J.; Khan, F. Non-pharmacological interventions for chronic pain in multiple sclerosis. Cochrane Database Syst. Rev. 2018, 12, CD012622. [CrossRef]

16. Jawahar, R.; Oh, U.; Yang, S.; Lapane, K.L. A systematic review of pharmacological pain management in multiple sclerosis. Drugs 2013, 73, 1711-1722. [CrossRef]

17. Rieckmann, P.; Centonze, D.; Elovaara, I.; Giovannoni, G.; Havrdová, E.; Kesselring, J.; Kobelt, G.; Langdon, D.; Morrow, S.A.; Oreja-Guevara, C.; et al. Unmet needs, burden of treatment, and patient engagement in multiple sclerosis: A combined perspective from the MS in the 21st Century Steering Group. Mult. Scler. Relat. Disord. 2018, 19, 153-160. [CrossRef]

18. Řasová, K.; Freeman, J.; Cattaneo, D.; Jonsdottir, J.; Baert, I.; Smedal, T.; Romberg, A.; Feys, P.; Alves-Guerreiro, J.; Habek, M.; et al. Content and Delivery of Physical Therapy in Multiple Sclerosis across Europe: A Survey. Int. J. Environ. Res. Public Health 2020, 17, 886. [CrossRef]

19. Espejo-Antúnez, L.; Tejeda, J.F.-H.; Albornoz-Cabello, M.; Rodríguez-Mansilla, J.; de la Cruz-Torres, B.; Ribeiro, F.; Silva, A.G. Dry needling in the management of myofascial trigger points: A systematic review of randomized controlled trials. Complement. Ther. Med. 2017, 33, 46-57. [CrossRef]

20. Gattie, E.; Cleland, J.A.; Snodgrass, S. The Effectiveness of Trigger Point Dry Needling for Musculoskeletal Conditions by Physical Therapists: A Systematic Review and Meta-analysis. J. Orthop. Sports Phys. Ther. 2017, 47, 133-149. [CrossRef]

21. Salom-Moreno, J.; Sánchez-Mila, Z.; Ortega-Santiago, R.; Palacios-Ceña, M.; Truyol-Domínguez, S.; Fernández-de-las-Peñas, C. Changes in spasticity, widespread pressure pain sensitivity, and baropodometry after the application of dry needling in patients who have had a stroke: A randomized controlled trial. J. Manip. Physiol. Ther. 2014, 37, 569-579. [CrossRef] [PubMed]

22. Mendigutia-Gómez, A.; Martín-Hernández, C.; Salom-Moreno, J.; Fernández-de-las-Peñas, C. Effect of Dry Needling on Spasticity, Shoulder Range of Motion, and Pressure Pain Sensitivity in Patients With Stroke: A Crossover Study. J. Manip. Physiol. Ther. 2016, 39, 348-358. [CrossRef] [PubMed]

23. Valencia-Chulián, R.; Heredia-Rizo, A.M.; Moral-Munoz, J.A.; Lucena-Anton, D.; Luque-Moreno, C. Dry needling for the management of spasticity, pain, and range of movement in adults after stroke: A systematic review. Complement. Ther. Med. 2020, 52, 102515. [CrossRef] [PubMed]

24. Boyce, D.; Wempe, H.; Campbell, C.; Fuehne, S.; Zylstra, E.; Smith, G.; Wingard, C.; Jones, R. Adverse Events Associated With Therapeutic Dry Needling. Int. J. Sports Phys. Ther. 2020, 15, 103-113. [CrossRef]

25. Ghannadi, S.; Shariat, A.; Ansari, N.N.; Tavakol, Z.; Honarpishe, R.; Dommerholt, J.; Noormohammadpour, P.; Ingle, L. The Effect of Dry Needling on Lower Limb Dysfunction in Poststroke Survivors. J. Stroke Cerebrovasc. Dis. 2020, 29, 104814. [CrossRef]

26. Blasini, M.; Peiris, N.; Wright, T.; Colloca, L. The Role of Patient-Practitioner Relationships in Placebo and Nocebo Phenomena. Int. Rev. Neurobiol. 2018, 139, 211-231. [CrossRef]

27. Quispe-Cabanillas, J.G.; Damasceno, A.; von Glehn, F.; Brandão, C.O.; Damasceno, B.P.; Silveira, W.D.; Santos, L.M.B. Impact of electroacupuncture on quality of life for patients with Relapsing-Remitting Multiple Sclerosis under treatment with immunomodulators: A randomized study. BMC Complement. Altern. Med. 2012, 12, 209. [CrossRef]

28. Charles, D.; Hudgins, T.; MacNaughton, J.; Newman, E.; Tan, J.; Wigger, M. A systematic review of manual therapy techniques, dry cupping and dry needling in the reduction of myofascial pain and myofascial trigger points. J. Bodyw. Mov. Ther. 2019, 23, 539-546. [CrossRef] 
29. Luque-Moreno, C.; Granja-Domínguez, A.; Heredia-Rizo, A.M.; Lucena-Anton, D.; Galán-Mercant, A.; Moral-Munoz, J.A. Multiple sclerosis treatment: Dry needling, new therapeutic frontiers. J. Adv. Health Care 2020, 2, 1-5. [CrossRef]

30. Cagnie, B.; Dewitte, V.; Barbe, T.; Timmermans, F.; Delrue, N.; Meeus, M. Physiologic effects of dry needling topical collection on myofascial pain. Curr. Pain Headache Rep. 2013, 17, 348. [CrossRef]

31. Castro Sánchez, A.M.; García López, H.; Fernández Sánchez, M.; Pérez Mármol, J.M.; Aguilar-Ferrándiz, M.E.; Luque Suárez, A.; Matarán Peñarrocha, G.A. Improvement in clinical outcomes after dry needling versus myofascial release on pain pressure thresholds, quality of life, fatigue, pain intensity, quality of sleep, anxiety, and depression in patients with fibromyalgia syndrome. Disabil. Rehabil. 2019, 41, 2235-2246. [CrossRef] [PubMed]

32. Sarmiento-Hernández, I.; Pérez-Marín, M.D.L.Á.; Nunez-Nagy, S.; Pecos-Martín, D.; Gallego-Izquierdo, T.; Sosa-Reina, M.D. Effectiveness of Invasive Techniques in Patients with Fibromyalgia: Systematic Review and Meta-Analysis. Pain Med. 2020. [CrossRef] [PubMed]

33. Chan, A.W.; Tetzlaff, J.M.; Gøtzsche, P.C.; Altman, D.G.; Mann, H.; Berlin, J.A.; Dickersin, K.; Hróbjartsson, A.; Schulz, K.F.; Parulekar, W.R.; et al. SPIRIT 2013 explanation and elaboration: Guidance for protocols of clinical trials. BMJ 2013, 346, e7586. [CrossRef] [PubMed]

34. Polman, C.H.; Reingold, S.C.; Banwell, B.; Clanet, M.; Cohen, J.A.; Filippi, M.; Fujihara, K.; Havrdova, E.; Hutchinson, M.; Kappos, L.; et al. Diagnostic criteria for multiple sclerosis: 2010 Revisions to the McDonald criteria. Ann. Neurol. 2011, 69, 292-302. [CrossRef] [PubMed]

35. Kurtzke, J.F. Rating neurologic impairment in multiple sclerosis: An expanded disability status scale (EDSS). Neurology 1983, 33, 1444-1452. [CrossRef] [PubMed]

36. Tang, L.; Yan, L.; Qiang-Min, H.; Yang, Y. Dry needling at myofascial trigger points mitigates chronic post-stroke shoulder spasticity. Neural Regen. Res. 2018, 13, 673-676. [CrossRef]

37. Streitberger, K.; Kleinhenz, J. Introducing a placebo needle into acupuncture research. Lancet 1998, 352, 364-365. [CrossRef]

38. Řasová, K.; Martinková, P.; Soler, B.; Freeman, J.; Cattaneo, D.; Jonsdottir, J.; Smedal, T.; Romberg, A.; Henze, T.; Santoyo-Medina, C.; et al. Real-world goal setting and use of outcome measures according to the international classification of functioning, disability and health: A european survey of physical therapy practice in multiple sclerosis. Int. J. Environ. Res. Public Health 2020, 17, 4774. [CrossRef]

39. Hochsprung, A.; Heredia-Camacho, B.; Castillo, M.; Izquierdo, G.; Escudero-Uribe, S. Clinical validity of the quantitative gait variables in patients with multiple sclerosis. A comparison of the Timed 25-foot Walk Test and the GAITRite (R) Electronic Walkway system. Rev. Neurol. 2014, 59, 8-12.

40. Ares-Benítez, I.; Luque-Moreno, C.; Alonso-Enríquez, L.; Moral-Munoz, J.A.; Lucena-Anton, D.; Izquierdo-Ayuso, G.; Granja-Domínguez, A. Physical therapy and Spanish dance in the reeducation of gait disturbances in multiple sclerosis: A case report. Rev. Cient. Soc. Esp. Enferm. Neurol. 2020. [CrossRef]

41. Gijbels, D.; Eijnde, B.O.; Feys, P. Comparison of the 2- and 6-minute walk test in multiple sclerosis. Mult. Scler. J. 2011, 17, 1269-1272. [CrossRef] [PubMed]

42. Ghotbi, N.; Nakhostin Ansari, N.; Naghdi, S.; Hasson, S. Measurement of lower-limb muscle spasticity: Intrarater reliability of Modified Modified Ashworth Scale. J. Rehabil. Res. Dev. 2011, 48, 83-88. [CrossRef]

43. Banos, O.; Moral-Munoz, J.A.; Diaz-Reyes, I.; Arroyo-Morales, M.; Damas, M.; Herrera-Viedma, E.; Hong, C.S.; Lee, S.; Pomares, H.; Rojas, I.; et al. MDurance: A novel mobile health system to support trunk endurance assessment. Sensors 2015, 15, 13159-13183. [CrossRef] [PubMed]

44. Molina-Molina, A.; Ruiz-Malagón, E.J.; Carrillo-Pérez, F.; Roche-Seruendo, L.E.; Damas, M.; Banos, O.; García-Pinillos, F. Validation of mDurance, A Wearable Surface Electromyography System for Muscle Activity Assessment. Front. Physiol. 2020, 11, 606287. [CrossRef]

45. Hermens, H.; Freriks, B.; Disselhorst-Klug, C.; Rau, G. Development of recommendations for SEMG sensors and sensor placement procedures. J. Electromyogr. Kinesiol. 2000, 10, 361-374. [CrossRef]

46. Beretta-Piccoli, M.; Cescon, C.; Barbero, M.; Villiger, M.; Clijsen, R.; Kool, J.; Kesselring, J.; Bansi, J. Upper and lower limb performance fatigability in people with multiple sclerosis investigated through surface electromyography: A pilot study. Physiol. Meas. 2020, 41, 025002. [CrossRef]

47. Fernández-de-Las-Peñas, C.; Ortega-Santiago, R.; Ortíz-Gutiérrez, R.; Caminero, A.B.; Salom-Moreno, J.; Arendt-Nielsen, L. Widespread pressure pain hypersensitivity in patients with multiple sclerosis with and without pain as sign of central sensitization. Clin. J. Pain 2015, 31, 66-72. [CrossRef] 
48. Fernández, O.; Fernández, V.; Baumstarck-Barrau, K.; Muñoz, L.; Gonzalez Alvarez, M.d.M.; Arrabal, J.C.; León, A.; Alonso, A.; López-Madrona, J.C.; Bustamante, R.; et al. Validation of the spanish version of the Multiple Sclerosis International Quality of Life (Musiqol) questionnaire. BMC Neurol. 2011, 11, 127. [CrossRef]

49. Apti, A.; Akalan, N.E.; Kuchimov, S.; Özdinçler, A.R.; Temelli, Y.; Nene, A. Plantar flexor muscle weakness may cause stiff-knee gait. Gait Posture 2016, 46, 201-207. [CrossRef]

50. Lencioni, T.; Jonsdottir, J.; Cattaneo, D.; Crippa, A.; Gervasoni, E.; Rovaris, M.; Bizzi, E.; Ferrarin, M. Are Modular Activations Altered in Lower Limb Muscles of Persons with Multiple Sclerosis during Walking? Evidence from Muscle Synergies and Biomechanical Analysis. Front. Hum. Neurosci. 2016, 10, 620. [CrossRef]

Publisher's Note: MDPI stays neutral with regard to jurisdictional claims in published maps and institutional affiliations.

(C) 2020 by the authors. Licensee MDPI, Basel, Switzerland. This article is an open access article distributed under the terms and conditions of the Creative Commons Attribution (CC BY) license (http://creativecommons.org/licenses/by/4.0/). 